\title{
Position Tracking for Passive UHF RFID Tags with the Aid of a Scanned Array
}

\author{
Antonios Bouzakis · Ludger Overmeyer
}

Received: 26 March 2013/Accepted: 30 May 2013/Published online: 23 June 2013

(c) The Author(s) 2013. This article is published with open access at Springerlink.com

\begin{abstract}
Thanks to the proliferation of radio frequency identification systems (RFID), applications have emerged concerning positioning techniques for inexpensive passive RFID tags. The most accurate approaches for tracking the tag's position, deliver precision in the order of $20 \mathrm{~cm}$ over a range of a few meters and require moving parts in a predefined pattern (mechanical antenna steering), which limits their application. Herein, we introduce an RFID tag positioning system that utilizes an active electronically-steered array, based on the principles of modern radar systems. We thoroughly examine and present the main attributes of the system with the aid of an finite element method simulation model and investigate the system performance with far-field tests. The demonstrated positioning precision of $1.5^{\circ}$, which translates to under $1 \mathrm{~cm}$ laterally for a range of a few meters can be helpful in applications like mobile robot localization and the automated handling of packaged goods.
\end{abstract}

Keywords RFID - Tag positioning - Scanned-array ·

Location awareness

\section{Introduction}

The radio frequency identification (RFID) is an automatic data collection technology mainly used for object identification and tracking [1-3]. A typical RFID system comprises

A. Bouzakis $(\bowtie) \cdot$ L. Overmeyer

Institute of Transport and Automation Technology, Leibniz

Universität Hannover, An der Universität 2, Garbsen,

Niedersachsen 30823, Germany

e-mail: antonios.bouzakis@ita.uni-hannover.de

L. Overmeyer

e-mail: ludger.overmeyer@ita.uni-hannover.de a reader module, which modulates data and commands into an RF signal, along with an antenna for the signal transmission. Passive RFID tags when located inside the reader's electromagnetic field acquire energy with the aid of their built-in antenna by means of inductive or radiative coupling. The acquired energy is subsequently used to power up an IC chip with integrated memory attached to the tag antenna [4]. The increasing need for higher bit rates and longer range for supply chain management dictated higher frequencies. These prerequisites provided the impetus for the vast deployment of ultra high frequency systems (UHF, 860-960 MHz) operating under the backscatter principle, where electromagnetic waves propagating between the reader and the tag antenna are employed to power up the tags. This operation can be summarized by the forward link (reader to tag), the re-modulation of the received signal by the chip and the reverse link (tag to reader) [5].

\section{State of the Art}

The operation of ultra-high frequency (UHF) systems is based on electromagnetic waves propagating between antennas. The power of a transmitted signal as detected by a receiver, considering perfectly matched antennas and no polarization mismatches, can be mathematically expressed with Friss equation as

$P_{R X}=P_{T X} \cdot G_{T X} \cdot G_{R X} \cdot \frac{\lambda^{2}}{(4 \pi r)^{2}}$

where $P_{T X}$ is the transmitted power, $P_{R X}$ the power detected at the receiver, $r$ is the distance between the two antennas and $G_{T X}$ and $G_{R X}$ are the gain of the transmitting and the receiving antenna respectively. However, if we solve Friss 
equation for the distance $r$ and try to calculate the distance between the reader antenna and an RFID tag, we will acquire merely a rough positioning estimation mainly because the RFID signal is extremely narrowband and thus suffers from multipath. The same stands for time of travel (TOF) measurements. Nowadays common positioning techniques are based on location fingerprinting and normally make use of a Kalman filter, or lie on a predefined antenna radiation pattern and mechanical steering to define the relative angle between the reader antenna and the tag.

In [6] a technique is described for determining the position of UHF tags with the aid of several antennas, each assigned a spatial probabilistic distribution of positive tag encounter. These antennas are mechanically rotated to scan a room populated with several tags. In this way, a map is created that indicates the tag positions with the highest probability. The same methodology is used in [7], where a similar system is deployed on a mobile robot for localization and mapping purposes. These systems offer a rather coarse accuracy of $50-100 \mathrm{~cm}$. In [8], a positioning methodology based on the response rate of UHF tags is described. Tags located closer to the reader antenna respond more frequently than distant ones. Following the same principles a reader module is deployed on a moving vehicle to detect its relative position from stationary tags [9]. However, no useful information on the accuracy of theses systems is provided. In [10] and [11] a mobile robot is described, featuring an onboard UHF reader module. The reader antenna is rotated in space and the tag positive encounters are collected. The position of the tags is estimated with the aid of the antenna radiation pattern. While the antenna is rotating, at some specific angle the tags enter the main lobe of the reader antenna and at some angle later leave this zone. Assuming a symmetrical radiation pattern the angle between the reader antenna and the tag can be estimated with an accuracy of $6^{\circ}-7^{\circ}$ deviation.

In [12] the same methodology is followed. However, the reader antenna is fixed on the end-effector of a robotic manipulator. Firstly, the relative angle between the reader antenna and the tag is estimated by rotating the antenna and afterwards the manipulator moves the antenna to a different position in space and rotates it again, thus estimating a second relative angle. In this way, the tag position on a $2 \mathrm{D}$ Cartesian coordinate system can be predicted with the aid of a triangulation algorithm. The illustrated maximum accuracy for this system lies at $25 \mathrm{~cm}$. In [13] the position of a mobile robot with an onboard reader is estimated with the aid of tags integrated into the floor at predefined positions. To estimate its position, the reader acquires and measures the backscattered signal from the several tags and uses an weighted mean of the acquired received signal strength indicator (RSSI) values. The accuracy of the presented systems lies at 30-80 cm. An excellent technique for positioning passive UHF tags is described in [14], using holographic localization. The presented accuracy lies at around $20 \mathrm{~cm}$, it is however rather time consuming and requires high computational capacities.

\section{Tag Positioning with the Aid of Beam-Steering}

Our approach focused on rather estimating the angular position of a tag relative to the reader antenna based on the principles of active electronically-steered radar systems. The herein introduced tracking system comprises a controlled reception pattern antenna (CRPA) array. It can be combined with any common commercial UHF reader module that delivers an RSSI value and is easy to implement for typical warehousing applications.

The operation principle of the developed tracking system lies upon electronically adjusting the shape of the radiation pattern of the reader antenna, in such way as to divert more power towards a desired direction in space. This evidently requires a directive antenna that mainly radiates power within a narrow area (main lobe). It is apparent that a tag located in the direction of the main lobe will receive more power and is therefore more likely to respond back to the reader. Moreover, the amplitude of the signal backscattered from a tag that is located closer to the center of the main lobe will be higher than for a tag that is further away from the center. We can therefore track the angular tag position with respect to the reader antenna by electronically adjusting the direction of the main lobe and collecting the positive tag responses along with the RSSI, which is the amplitude of the backscattered signal from the tag, as measured at the reader module. The power of the backscattered tag signal received by a monostatic antenna in an arbitrary propagation environment can be written in form of a classical radar equation as $[15,16]$ :

$P_{R X, \text { reader }}=P_{T X, \text { reader }} \cdot G_{\text {reader }}^{2} \cdot G_{\text {tag }}^{2} \cdot \frac{\lambda^{4}}{(4 \pi r)^{4}} \cdot m_{\text {eff }}$

where $P_{T X, \text { reader }}$ is the power transmitted by the reader antenna, $G_{\text {reader }}$ is the gain of the reader antenna, $r$ is the distance between the reader and the tag antenna, $G_{\text {tag }}$ is the gain of the tag antenna and $m_{\text {eff }}$ is the modulation efficiency of the tag IC. It is apparent from Eq. 2 that the power of the tag signal is proportional to the squared gain of the reader antenna. Therefore, by spatially varying the gain of the reader antenna in a predefined manner and measuring the power of the backscattered tag signal, we can estimate the angular position of the tag with respect to the reader antenna as illustrated in Fig. 1.

It is apparent that to accomplish this, we need to precisely determine the reference point of measurement for 


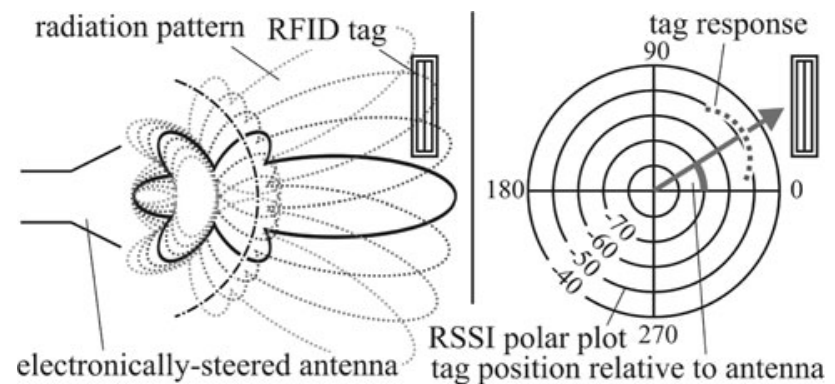

Fig. 1 Principle of tag tracking with the aid of beam-steering [17]

both antennas, which is called the antenna phase. For simple antenna structures, like the typical dipole antenna of a tag, it is safe to assume that the antenna phase will be the center of the antenna geometry. CRPAs may however feature more complex geometries, for which case mathematical models have been introduced and tested for defining the position of the antenna phase [18, 19]. An excellent study on occurring offsets for the antenna phase of a CRPA array with beam steering-adaptive forming capabilities is presented in [20]. Note that the majority of electronically-steered radar systems employ antenna arrays with several elements. Although, there exist various techniques for steering the beam, the most widely used include feeding each array element with a signal of different amplitude or different phase. A combination of both is also common.

\section{Development of the Scanned Array}

Electronically-steered antenna arrays typically feature several elements, more than often aligned in two dimensions. The herein introduced system was designed to feature solely three elements horizontally aligned and spaced $160 \mathrm{~mm}$ apart, resulting in approximately half wavelength spacing $(\approx 173 \mathrm{~mm}$ at $866 \mathrm{MHz}$ ). It is safe to assume that the antenna phase of the developed tracking system is located at the geometrical center of the array. In other words the antenna phase lies at the center of the middle element, which was used as the reference point for the measurements. As depicted in Fig. 2 once the elements are chosen, the construction is relatively simple.

Note that we can achieve higher directivity by employing more elements or simply by increasing the spacing between the existing ones. This would however increase the size of the antenna array and hence also the boundary of the near-field. Considering that the introduced tracking principle is based on the pattern formation, it is essential to operate in the antenna far-field. Therefore, we would like to keep the near-field boundary as close to the antenna as possible, to be able to detect the position of less

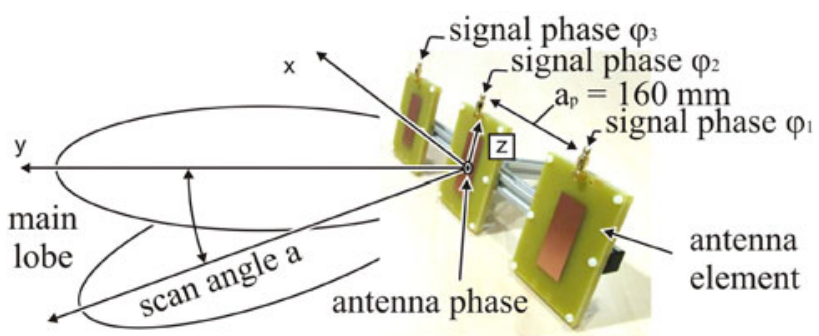

Fig. 2 The developed antenna array

distant tags. An overview on the near-field and far-field regions of an arbitrary antenna can be found in [21]. Accordingly, the far-field boundary condition,

$R=\frac{2 \cdot D^{2}}{\lambda}$

which is critical for the pattern formation was taken into account. The far-field region for the developed tracking system begins at $980 \mathrm{~mm}$.

To maintain simplicity, we chose to use the same signal amplitude for all the elements and employ a phase controller for each element to steer the beam. Hence, the direction of the main lobe denoted as the scan angle a, is adjusted by using a predefined combination for the phases of the signal fed to each element. This operation is described in Sects. 4.2 and 4.3.

\subsection{Development of the Antenna Elements}

For the elements of the antenna array, we developed an aperture coupled microstrip antenna, known for being highly directive and especially suitable for pattern forming [21]. The geometrical properties of this vertically polarized antenna, are illustrated in Table 1.

The behavior of the antenna return loss (RL) over the frequency was calculated through an FEM analysis and was

Table 1 Dimensions of the developed aperture coupled antenna

\begin{tabular}{lll}
\hline Symbol & Dimension & Designation \\
\hline$\varepsilon_{r}$ & 4.25 & Relative permittivity \\
$f_{w}$ & $5 \mathrm{~mm}$ & Width of the feed line \\
$w_{a}$ & $5 \mathrm{~mm}$ & Aperture width \\
$w_{s}$ & $90 \mathrm{~mm}$ & Substrate width \\
$w_{p}$ & $30 \mathrm{~mm}$ & Patch width \\
$t_{1}$ & $5 \mathrm{~mm}$ & Feed line substrate thickness \\
$t_{2}$ & $5 \mathrm{~mm}$ & Patch substrate thickness \\
$l_{a}$ & $30 \mathrm{~mm}$ & Aperture length \\
$l_{s}$ & $120 \mathrm{~mm}$ & Substrate length \\
$l_{p}$ & $73 \mathrm{~mm}$ & Patch length \\
\hline
\end{tabular}


verified with the aid of a vector network analyzer (Rohde \& Schwarz FSH3 plus the FSH Z2). As expected the antenna features a $-10 \mathrm{~dB}$ bandwidth of $30 \mathrm{MHz}(858-888 \mathrm{MHz}$ ) that completely covers the European RFID band, within which all tests were carried out. Such antennas principally suffer from narrow bandwidth [22]. However, enhanced bandwidth can be achieved through a stacked configuration $[23,24,25]$, for covering the entire international RFID band (860-960 MHz). The developed antenna, as well as the RL are presented in Fig. 3.

\subsection{Simulation of the Scanned Array}

The steering of the beam is carried out by controlling the phase of the signal on each antenna element, basically by adjusting the phase of the first element $\phi_{1}$ to lead the phase of the middle element $\phi_{2}$ by several degrees and simultaneously the phase of the last element $\phi_{3}$ to lag the phase of the middle element $\phi_{2}$ by the same amount according to the equation:

$\phi_{1}-\phi_{2}=\phi_{2}-\phi_{3}$

This operation is illustrated with the aid of a simulation model in Fig. 4. For $\phi_{2}=0$, we simply need to assign opposite numbers to $\phi_{1}$ and $\phi_{3}$ to adjust the scan angle a, thus steering the beam on the $\mathrm{x}-\mathrm{y}$ plane.

\subsection{Development of the Steering Controller}

It is clear that we need to split the signal going out of the reader module and then feed it to each antenna element after applying the respective phase shift. Therefore, a 3-way $(-4.8 \mathrm{~dB})$ splitter/combiner $\mathrm{SCN}-3-13+$ from minicircuits was employed to distribute the signal to the elements and furthermore gather and combine the tag signal received by each antenna element. The employed phase shifters JSPHS 1000+ from minicircuits are analog controlled low-loss devices offering an arbitrary phase shift of maximum $190^{\circ}$ at $866 \mathrm{MHz}$. Their operation is based on the varactor diode principle, where a DC voltage is utilized to control the phase of the signal as described in [26]. Typical values for the insertion loss do not exceed $-1.2 \mathrm{~dB}$
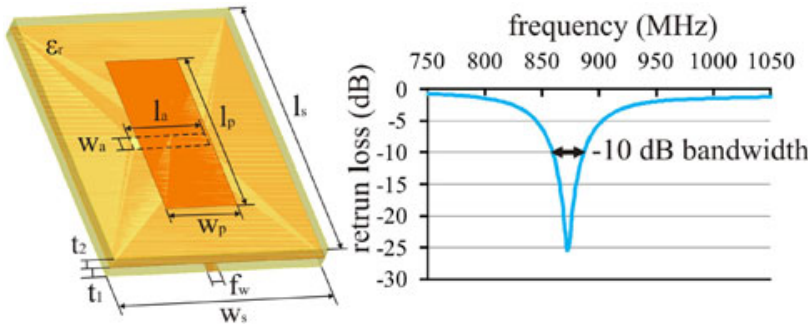

Fig. 3 Developed aperture-coupled microstrip antenna [17]

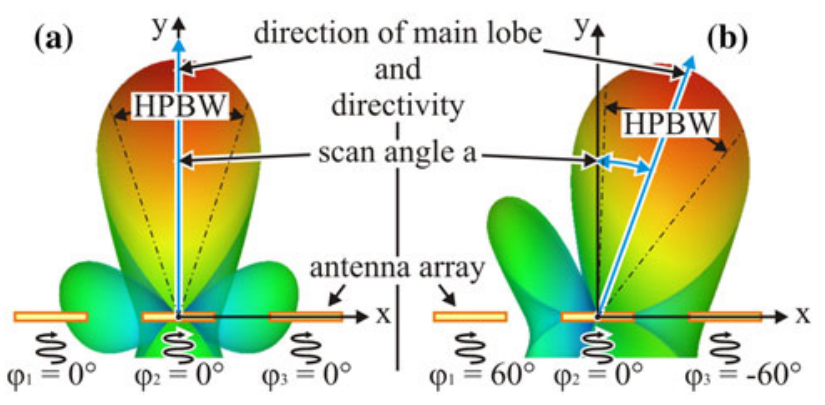

Fig. 4 Simulation model of the developed scanned array [17]

and remain almost constant over the entire phase span. A series of two phase shifters was used for each element to achieve a complete $360^{\circ}$ phase shift as illustrated in Fig. 5 . The maximum phase deviation of the signal traveling through the splitter/combiner and a series of two phase shifters was measured with the aid of the vector network analyzer and found to be $\pm 1^{\circ}$. This deviation eventually affects the overall accuracy of the system. Finally, the A/D (analog-to-digital) converter NI 9264 from National Instruments was employed to apply the necessary voltage for adjusting the signal phase. The steering controller is presented in Fig. 5.

Note that the overall cost of the system does not exceed the price of a commercial RFID reader module. The antenna elements are low-cost PCBs and the splitter/combiner as well as the phase shifters are off-the-shelf surfacemount devices. The selected A/D converter is the most expensive part of this particular system. It does however provide accuracy higher than the required for adjusting the DC voltage. Note that more cost-efficient A/D converters suited for this application are commercially available.

\section{Attributes of the Developed Tracking System}

In Fig. 4 it can be clearly observed that for a specific phase offset between the radiating elements, the direction of the main lobe (scan angle a) is shifted in the $x-y$ plane. It should be noted that if a tag is located outside of the

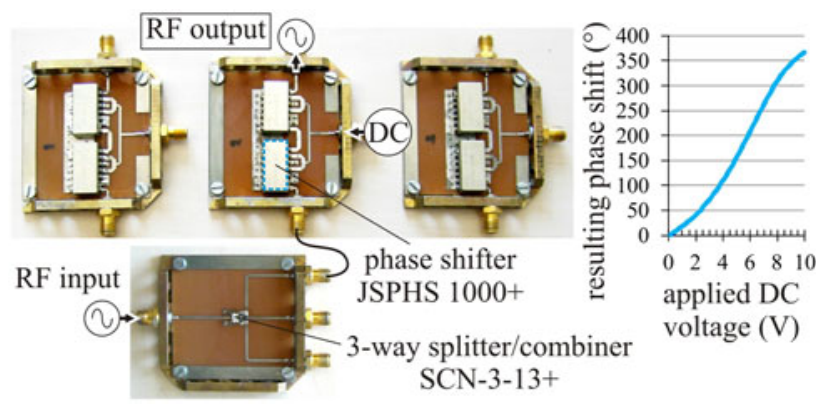

Fig. 5 Steering controller for the scanned array [17] 
HPBW (half power beam width) it does not necessarily mean that the power will not suffice for the RFID communication to take place. The HPBW shows how densely the power is distributed along the direction of the main lobe and is in this case a figure of merit illustrating the expected trace width for a single measurement. As further on demonstrated the width of the acquired trace is dependent upon the distance between the tag and the antenna array.

Considering that the introduced principle for tracking the position of a UHF RFID tag with the aid of beamsteering is based on the pattern formation, it is essential for the tracking system to provide consistent pattern characteristics over a desired scan angle span. The scan angle itself is however not a controllable variable. The variables we can control for this system are the phases of the signal for each element $\left(\phi_{1}, \phi_{2}, \phi_{3}\right)$. To simplify calculations we considered assigning $\phi_{2}=0$ and selecting $\phi_{1}$ as the controlled variable. Consequently, $\phi_{3}$ is automatically defined through Eq. 4. In this case the behavior of the most important system characteristics with respect to the input variable $\phi_{1}$, as deriving from the FEM simulation is illustrated in Fig. 6.

The direction of the main lobe (scan angle a) is practically shifting linearly with the applied phase shift at the first element $\left(\phi_{1}\right)$ on a 3:1 ratio for a signal-phase span of $\left[-90^{\circ},+90^{\circ}\right]$. This eventually provides an effective scanangle span of a total $60^{\circ}$. Within this span the directivity yields a maximum of $5.57 \mathrm{dBi}$ when the main lobe is pointing at $0^{\circ}$ and a maximum of $5.3 \mathrm{dBi}$ at $\pm 30^{\circ}$. This antenna gain drop-off occurring when the main lobe shifts to either side will eventually alter the outcome of the measurement and needs therefore to be compensated for. Furthermore, the HPBW varies considerably from $36^{\circ}$ to $42^{\circ}$ within the scan-angle span and in combination with the power gain drop-off will also affect the results and needs to be taken into account.

\section{Positioning Algorithm and Performance}

If we consider the model presented in Fig. 4, it is clear that to estimate the position of a receiver located at an arbitrary position in front of the scanned array, we need to determine the scan angle a, wherefore the power of the received

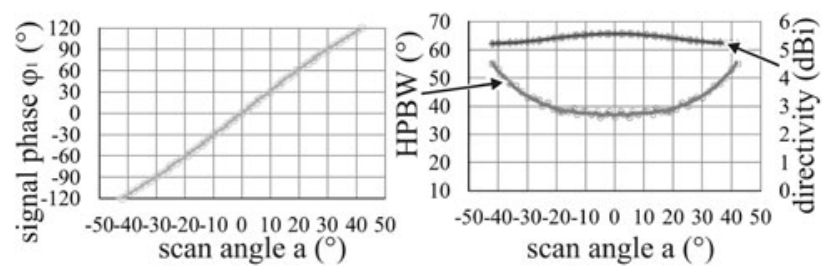

Fig. 6 Attributes of the developed scanned array [17] signal maximizes. In other words, considering that all other variables are kept constant, we want to determine the scan angle a, for which the receiver is illuminated by the maximum gain of the transmitter. To achieve this we simulated a receiver placed at different scan angles in the far-field of the scanned array within $\left[0,+30^{\circ}\right]$ and acquired the values of the gain of the scanned array that the receiver detects for different values of the signal phase $\phi_{1}$.

Furthermore, as illustrated in Fig. 7 we measured these values with the aid of a reference antenna, whose radiating characteristics are known, by transmitting a continuous wave from the scanned array and measuring its amplitude as received by the reference antenna. To calculate the gain of the scanned array with the aid of the power of the received signal, we used Eq. 1 multiplied by the transmission coefficient, so as to include the impedance mismatches:

$G_{T X}=\frac{P_{R X} \cdot(4 \pi r)^{2}}{P_{T X} \cdot G_{R X} \cdot \lambda^{2} \cdot \tau_{R X} \cdot \tau_{T X}}$

where $\tau_{R X}$ and $\tau_{T X}$ are the transmission coefficient of the receiving (reference) antenna and the transmitting (scanned array) antenna. The distance $r$ between the two antennas was $1.5 \mathrm{~m}$. The results from the simulation, as well as the measurement for the 31 individual positions are presented in Fig. 8, where all curves have been referenced to $0 \mathrm{dBi}$. Note that we sampled the values from the simulation for every $5^{\circ}$ of the signal phase $\phi_{1}$ within $\left[-120^{\circ},+120^{\circ}\right.$, however we sampled the received signal power during the measurement for every $1^{\circ}$ of $\phi_{1}$ to achieve better resolution. This means that each of the curves deriving form the simulation contains 48 measurement points, whereas each curve deriving from a measurement includes 240 points as presented in Fig. 8. For illustration purposes the red curve (left in each diagram) corresponds to a scan angle of $5^{\circ}$ and the blue (right in each diagram) to a scan angle of $30^{\circ}$.

Note that the first null positions appear steeper for the results from simulation than for the respective ones deriving from the measurement, mainly because in the simulation an infinite distance between the two antennas is assumed. If we increased the distance $r$ at the measurement, these minima

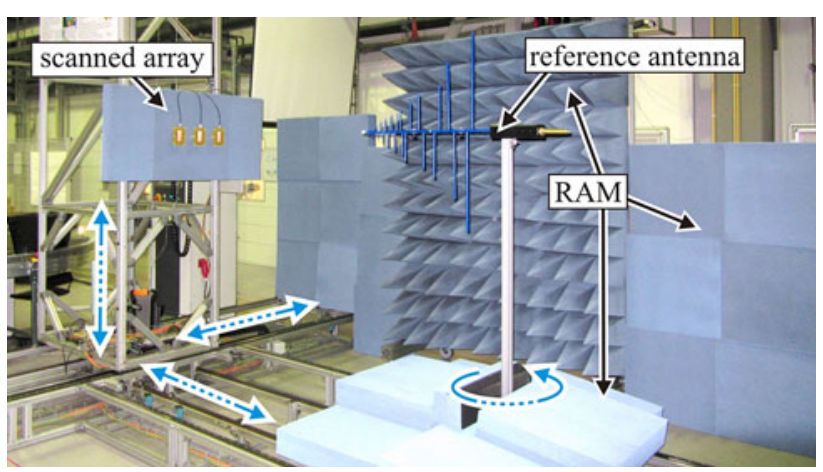

Fig. 7 Test bench for gain measurements 


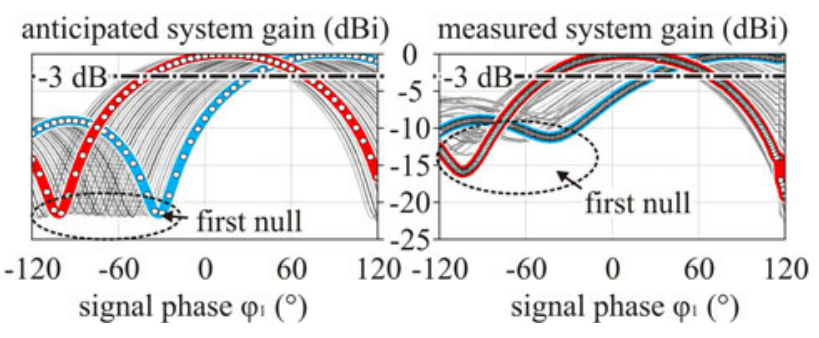

Fig. 8 Spatial gain distribution simulation and measurement

would also decrease significantly. From the diagrams in Fig. 8, we can now determine the signal phase $\phi_{1}$ for the maximum occurring gain value, for each of the 31 scan angles. We employed a quadratic squared regression, using all values higher than $-3 \mathrm{~dB}$ below the detected maximum (half power or higher). The result of this least square fitting is a smoothed curve, which features a maximum that should point to the actual maximum gain of the system. The results for both cases are presented in Fig. 9.

For the case of the simulation, where there are no distortions in the signal, the estimated scan angle $\alpha_{e s t}$ of the reference antenna is in good agreement with the actual one. It can be observed that at high scan angles, the estimated values are as expected about $0.5^{\circ}$ lower than the actual ones. However, the estimated scan angles deriving from the measurement $\alpha_{\text {meas }}$ deviate slightly more from the actual ones, mainly due manufacturing tolerances and need to be compensated for.

Furthermore, we measured the performance of the system for the negative scan angle values and used this information to calibrate the system's accuracy

\section{Measurements with Tags}

\subsection{Far-Field Measurements}

We have now established an effective angular span for the developed antenna array within $\left[-30^{\circ},+30^{\circ}\right]$. We

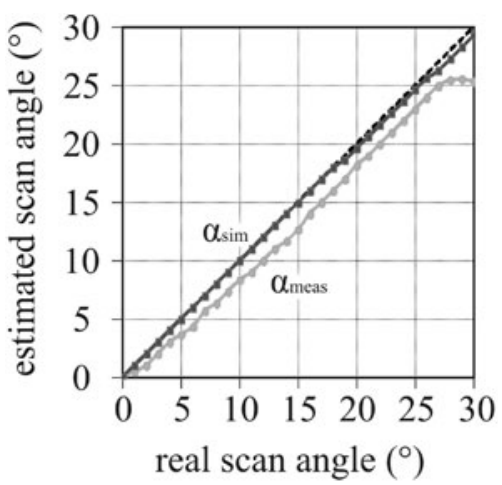

Fig. 9 Performance of the least square algorithm employed a commercial reader module IDS-DK-R902RD1 for testing the developed tracking system. The measurement basically included continuous interrogation for positive tag responses within the determined angular span. As soon as one scan angle was reviewed, the result (negative, or positive plus the RSSI) was stored and the beam was adjusted to the next scan angle. The time required for the total measurement is evidently dependent upon the interrogation rate of the reader module int $_{r}$ and for the primary tests summed up to $12 \mathrm{~s}$ for a $0.33^{\circ}$ scan angle resolution (20 inventory commands per second). However, note that this process can be accelerated either by employing a reader module that offers higher interrogation rate or by deteriorating the scan angle resolution. The measurement setup is presented in Fig. 10.

As illustrated in Fig. 10 the measurements were carried out with a commercial tag attached to a styrofoam piece and placed at predefined positions relative to the antenna phase. For adjusting the position, the same test bench with 4 degrees of freedom and $1 \mathrm{~mm}$ accuracy was used. Additionally, radiation absorbent material (RAM) was employed to minimize the interference due to reflected signals. To avoid polarization mismatches, the tag was fixed on a wooden pole in vertical alignment. A set of preprocessed results is presented in Fig. 11.

\subsection{Data Processing}

To estimate the angular tag position relative to the antenna, we firstly applied a least-square fitting with second order polynomials and estimated the scan angle $a_{e s t}$ for the maximum RSS indicator as depicted in Fig. 12. However, since the received backscattered signal is dependent upon the square of the gain of the scanned array, this time we filtered away all the values $6 \mathrm{~dB}$ lower than the detected maximum. As expected the result contained a systematic error which was defined by the system characteristics and

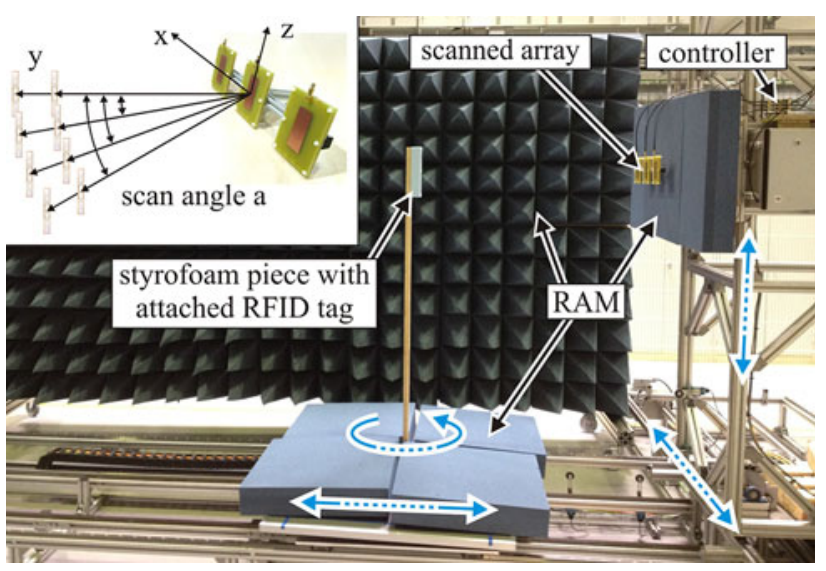

Fig. 10 Far-field measurement test bench [17] 


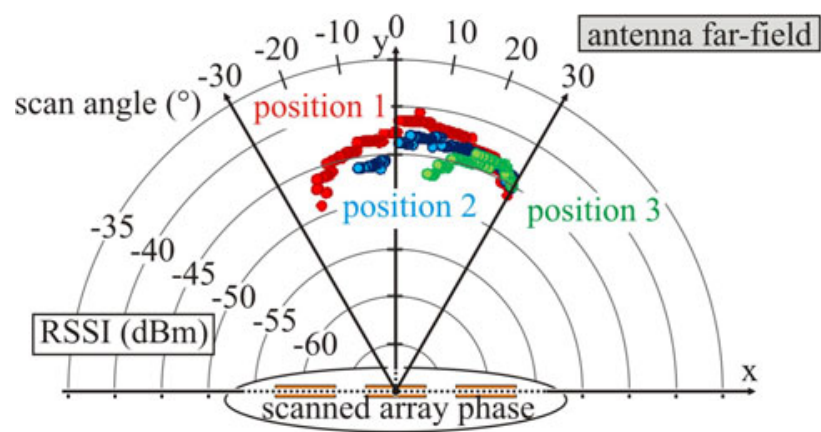

Fig. 11 Typical pre-processed results for tag tracking

could be alleviated with the aid of a compensation algorithm. Furthermore, a random error was included mainly due to the phase unbalance produced by the phase shifters and inserted into the signal of each antenna element, which is the key factor that defines the overall system accuracy.

To determine the accuracy of the tracking system we sampled all positions spaced $1^{\circ}$ apart for a $1,000 \mathrm{~mm}$ radius. Based on 30 acquired measurements for each position the mean value $\mu_{\text {mean }}$ was calculated as well as the standard deviation $s$ for $a_{e s t}$. As illustrated in Fig. 13 for the scan angle span between $\left[-28^{\circ},+28^{\circ}\right]$ the estimated angular position of the tag rises monotonically. The standard deviation remains under $0.5^{\circ}$ for all of the reviewed positions. This indicates that all of the estimated angular positions are practically located within $\pm 1^{\circ}$ from the real ones at a $95 \%$ confidence level. Using simple trigonometric equations we can interpret the angular deviation of $\pm 1^{\circ}$, to $\pm 17 \mathrm{~mm}$ in lateral positioning accuracy for the $1,000 \mathrm{~mm}$ distance between the reader and the tag.

However, the calculated positions contained a systematic error. It is apparent that when the beam shifts to the either side, the gain power drop-off combined with the changing size of the HPBW results in a decrease of the calculated position with regard to the real position. This can be corrected by applying a compensation algorithm,

$a_{\text {cor }}=c_{4} \cdot a_{e s t}^{4}+c_{3} \cdot a_{e s t}^{3}+c_{2} \cdot a_{e s t}^{2}+c_{1} \cdot a_{e s t}+c_{0}$

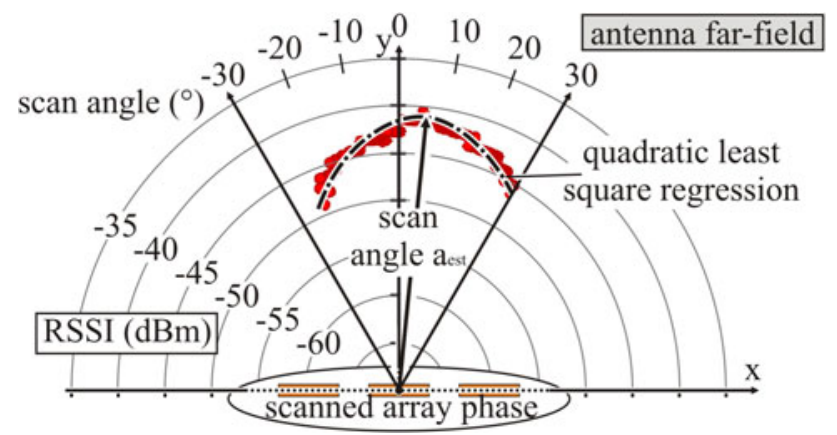

Fig. 12 Calculation of the angular tag position, scan angle $a_{\text {est }}$

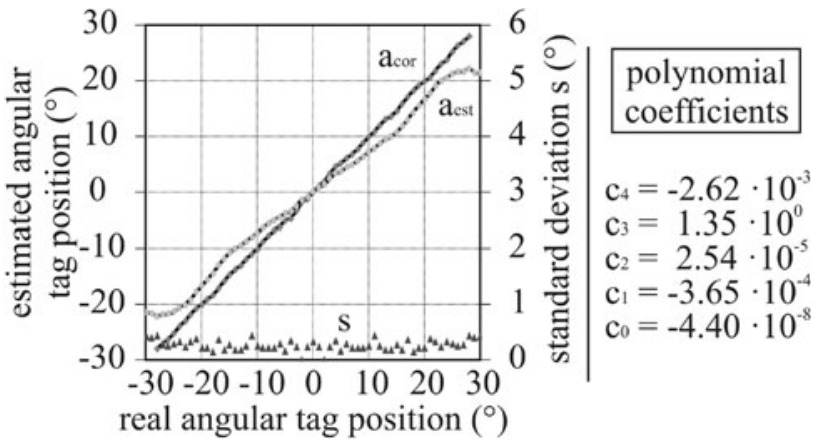

Fig. 13 System accuracy for a radius of $1000 \mathrm{~mm}$ according to [17]

where $a_{c o r}$ is the corrected angle between the tag and the antenna phase, $a_{e s t}$ is the estimated value after the least square fitting and the coefficients of the polynomials are defined so as to maximize accuracy while maintaining a monotonic behavior inside the scan angle span $\left[-28^{\circ},+28^{\circ}\right]$. The results are presented in Fig. 13.

Note that the compensation algorithm is employed to correct the systematic error that principally affects the mean value $\mu_{\text {mean }}$ and not the standard deviation $s$. It can be clearly observed that estimated tag positions after the correction algorithm is applied, are in good agreement with the real ones for the scan angle span of $\left[-28^{\circ},+28^{\circ}\right]$.

\subsection{Overall Accuracy}

Moreover, we investigated the accuracy of the system for different distances between the reader and the tag antenna. Herein, it should be noted that during the tests the ground reflection was not completely blocked. This allowed a more realistic illustration of the overall system performance, since in almost every application scenario the ground reflection is present. Consequently, there exist some blind spots where the path loss is exceedingly high for the reader-tag communication to occur. This is due to multipath and is primarily dependent upon the height of the antenna from the ground. However, in reality the effect of reflected signals is far more complex due to multiple reflections from objects, diffraction, shadowing, etc. [16]. This effect is also visible in Fig. 14.

We can see that the accuracy of the system remains almost constant with regard to the distance between the reader and the tag antenna, presenting a maximum deviation of $1.5^{\circ}-1.6^{\circ}$. The maximum deviation increases drastically when the multipath effect strongly attenuates the signal. It is therefore critical for the system to be able to recognize when the multipath effect is deteriorating the positioning accuracy. This can be achieved by reviewing the "completeness" of the acquired trace. By observing inside the trace it is clear that when strong reflections come 


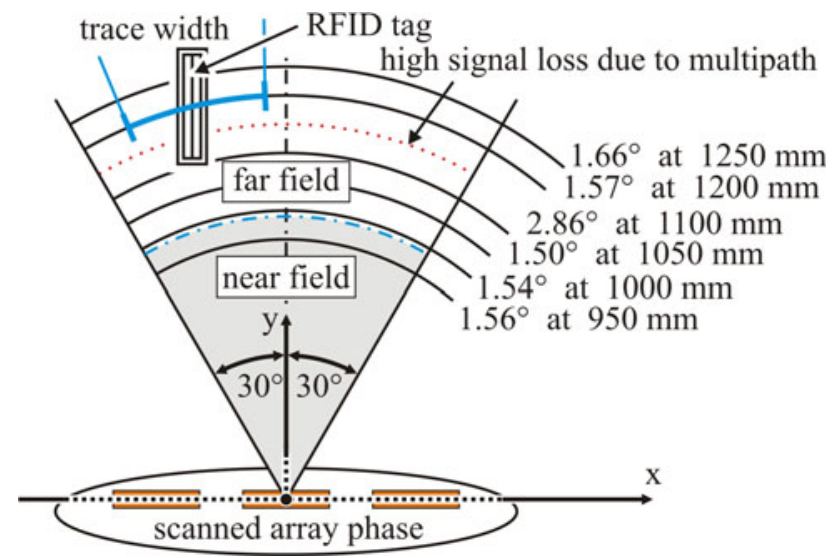

Fig. 14 Maximum positioning deviation for various tag distances [17]

into effect, for several scan angles the system fails to deliver the RSSI and thus the trace is incomplete.

\subsection{Tags in Motion}

From the previous analysis it is clear that the operation of the developed tracking system requires that both the tag and the reader antenna array are stationary. If we used a reader module offering an interrogation rate of 5 times higher than the employed one (around 100 responses per second) and consider deteriorating the scan angle resolution, for example one measurement every $0.66^{\circ}$, we could estimate the position of a tag within one second. In that case we could detect the position of a tag moving at a velocity within the accuracy of the system, which is expected to drop in the order of magnitude of $10 \mathrm{~cm}$.

We can however estimate the change of the radial position of the tag relative to the antenna array by using the width of the acquired trace. As previously discussed, the HPBW is herein used as a figure of merit to indicate how wide or narrow an acquired trace will be. The actual trace width is dependent upon the distance between the reader and the tag. In Fig. 15 the measured trace width is illustrated for diverse radial and angular positions.

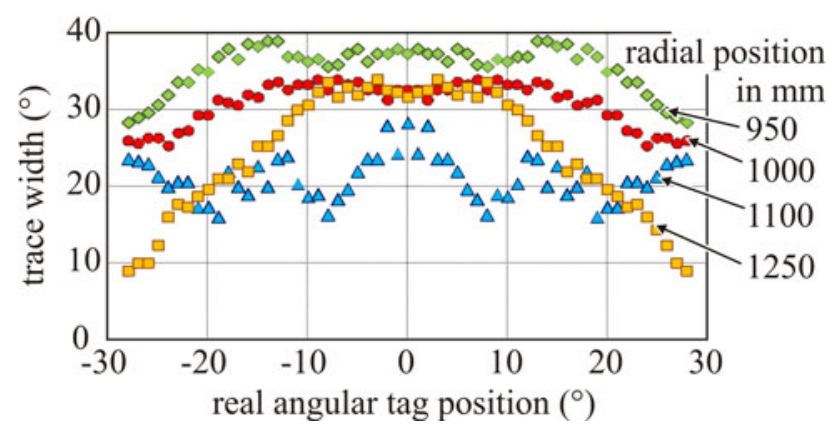

Fig. 15 Trace width for various tag positions as in [17]
As expected the trace width yields higher values meaning that it covers a wider angle span, when the tag is located closer to the reader antenna. This effect can assist in detecting slow moving tags even if the interrogation rate is low. In that case we can determine whether the tag is approaching or moving away from the antenna by continuously measuring the trace width. Although this principle does suffer from multipath, the positions where low accuracy is inevitable, can be determined by reviewing the "completeness" of the trace and be left out. For example if a tag is approaching the tracking system at a scan angle of $0^{\circ}$, the width of the trace is expected to be continuously rising. Contrariwise however, at a radius of $1,100 \mathrm{~mm}$ it will rapidly fall. In that case we can examine the completeness of the trace and eliminate it.

\section{Application Potential for Warehousing}

To illustrate how the developed UHF tracking system can be employed for the automated handling of packaged good, we mounted it on a suction gripper at the end effector of an industrial manipulator. In Fig. 16 we can observe a cardboard box with an attached UHF tag within the range of the tracking system. The algorithm used for determining the tag position on a $2 \mathrm{D}$ coordinate system is triangulation. After the tracking system scans the space from a predefined position, the end effector is relocated to a relatively distant one, again facing the same area of the conveyor where the tag was previously encountered.

By determining the estimated scan angle for each respective measurement point we can calculate the perpendicular distance of the tag $d_{t}$ from the line connecting the two measurement points according to the equation:

$d_{t}=\frac{h \cdot \sin a_{e s t, 1} \cdot \sin a_{e s t, 2}}{\sin \left(a_{e s t, 1}+a_{e s t, 2}\right)}$

In this way the position of one tag can be effectively determined with an accuracy defined by the estimated scan angles $a_{e s t}$. Note that although this approach appears to be in need of moving parts, thus resembling the one described in [12], we can employ a second scanned array located at the

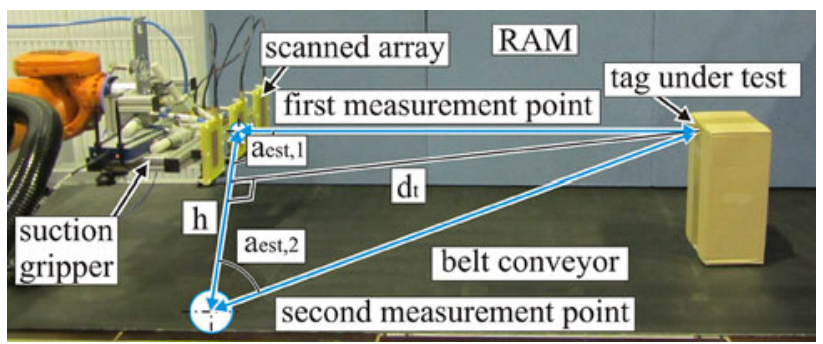

Fig. 16 Automated package tracking and handling 
distance from the first one, or develop a scanned array with more elements and use each time different combinations.

Moreover, an excellent example would be the navigation of automated guided vehicles (AGV) inside an indoor environment, where several tags are fixed at predefined positions. In that case the tracking system would be able to map the previously unknown environment and estimate the position of the AGV.

\section{Conclusion}

Herein an RFID positioning system utilizing beam forming was demonstrated. Such novel system can assist in maximizing the power absorbed by the tag by identifying its relative position and subsequently adjusting the beam direction without having to mechanically steer the antenna. This can be useful in applications where data have to be acquired and rewritten on the ICs memory, given that the tag's write sensitivity is lower than the read sensitivity, typically in the order of $3 \mathrm{dBm}$ [27]. The enhanced positioning precision of $<40 \mathrm{~mm}$ can be employed for determining the position of UHF tags e.g. by mobile robots for indoor localization and mapping, where the use of GPS is inhibited. Additionally, it can assist in applications where the handling of packaged products is carried out by automated manipulators. For this scenario a short break in the material flow is necessary to provide high precision. By conducting measurements from two different positions in space, it is possible to define the absolute position of a tag on a 2D Cartesian system through a triangulation algorithm. The width of the acquired trace can be employed to detect the movement of the tag. However, high interrogation rates are necessary. The use of even higher frequencies will allow the construction of a more complex tracking system featuring smaller antennas and increased directivity. Furthermore, at higher frequencies (shorter wavelengths) the electrical separation between a tag and reflecting objects increases [28], eventually leading to increased precision.

Acknowledgements We would like to thank the Hotoprint GmbH for producing the PCB-based antennas. This reasearch was sponsored by the German Research Foundation (DFG)

Open Access This article is distributed under the terms of the Creative Commons Attribution License which permits any use, distribution, and reproduction in any medium, provided the original author(s) and the source are credited.

\section{References}

1. J. Landt, The history of RFID, IEEE Potentials Vol. 24, No. 4, pp. 8-11, 2005.
2. R. Want, RFID explained : a primer on radio frequency identification technologies, Synthesis Lectures on Mobile and Pervasive Computing Vol. 1, No. 1, pp. 1-83, 2006.

3. L. Overmeyer, S. Vogeler, RFID: Grundlagen und Potenziale, Logistics Journal, Vol. S, pp. 1-12, 2005.

4. K. Finkenzeller, RFID Handbook: Radio-Frequency Identification Fundamentals and Applications, 2nd ed. Wiley, New York, 2004.

5. D.M. Dobkin, The RF in RFD: Passive UHF RFID in Practice, Elsevier, Burlington, 2007.

6. C. Alippi, D. Cogliati, G. Vanini, A statistical approach to localize passive RFIDs, in IEEE International Symposium on Circuits and Systems, 2006.

7. D. Hahnel, W. Burgard, D. Fox, K. Fishkin, M. Philipose, Mapping and localization with RFID technology, in IEEE International Conference on Robotics and Automation, Vol. 1, No. 1, pp. 1015-1020, 2004. doi:10.1109/ROBOT.2004.1307283.

8. J. Bing, K. Fishkin, S. Roy, M. Philipose, Unobtrusive long-range detection of passive RFID tag motion, in IEEE Transactions on Instrumentation and Measurement, Vol. 55, pp. 187-196, 2006.

9. P. Wilson, D. Prashanth, H. Aghajan, Utilizing RFID signaling scheme for localization of stationary objects and speed estimation of mobile objects, in IEEE International Conference on RFID, pp. 94-99, 2007.

10. S. Roh, H. Choi, 3-D tag-based RFID system for recognition of object, in IEEE Transactions on Automation Science and Engineering, Vol. 6, No. 1, pp. 55-65, 2009.

11. T. Hori, T. Wda, Y. Ota, N. Uchitomi, K. Mutsuura, H. Okada, A multi-sensing-range method for position estimation of passive RFID tags, in Networking and Communications, 2008. WIMOB '08. IEEE International Conference on Wireless and Mobile Computing, pp. 208-213, 2008.

12. M. Fujimoto, N. Uchitomi, A. Inada, T. Wada, K. Mutsuura, H. Okada, A novel method for position estimation of passive RFID tags; Swift Communication Range Recognition (S-CRR) method, in Global Telecommunications Conference (GLOBECOM 2010), 2010 IEEE, pp. 1-6, 2010. doi:10.1109/GLOCOM.2010.5683628.

13. Y. Park, J.W. Lee, S. Kim, Improving position estimation on RFID tag floor localization using RFID reader transmission power control, in IEEE International Conference on Robotics and Biomimetics, pp. 1716-1721, 2009.

14. R. Miesen, F. Kirsch, M. Vossiek, Holographic localization of passive UHF RFID transponders, in IEEE International Conference on RFID, pp. 32-37, 2011.

15. M.O. White, Radar cross-section: measurement, prediction and control, in Electronics Communication Engineering Journal, Vol. 10, No. 4, pp. 169-180, 1998. doi:10.1049/ecej:19980403.

16. P. Nikitin, K. Rao, Antennas and propagation in UHF RFID systems, in IEEE International Conference on RFID, pp. 277-288, 2008.

17. A. Bouzakis, L. Overmeyer, RFID tag positioning with the aid of an active electronically-steered array, in IEEE International Symposium on Personal, Indoor and Mobile Radio Communications PIMRC12, pp. 2483-2488, 2012.

18. J.P. Shang, D.M. Fu, Y.B. Deng, S. Jiang, Measurement of phase center for antenna with the method of moving reference point, in ISAPE 2008. 8th International Symposium on Antennas, Propagation and EM Theory, pp. 114-117, 2008. doi:10.1109/ISAPE. 2008.4735153.

19. Z. Pour, L. Shafai, Effect of primary feed polarization on phase centre location of parabolic reflector antennas, in Antennas and Propagation Society International Symposium (APSURSI), 2010 IEEE, pp. 1-4, 2010. doi:10.1109/APS.2010.5562186.

20. U.S. Kim, D. De Lorenzo, D. Akos, J. Gautier, P. Enge, J. Orr, Precise phase calibration of a controlled reception pattern GPS antenna for JPALS, in Position Location and Navigation 
Symposium, 2004. PLANS 2004, pp. 478-485, 2004. doi:10.1109/ PLANS.2004.1309032.

21. C.A. Balanis, Antenna Theory: Analysis and Design, 3rd ed. Wiley, New York, 2005.

22. D.M. Pozar, D.H. Schaubert, Microstrip Antennas: The Analysis and Design of Microstrip Antennas and Arrays, Wiley-IEEE Press, New York, 1995.

23. S. Targonski, R. Waterhouse, An aperture coupled stacked patch antenna with $50 \%$ bandwidth, in Antennas and Propagation Society International Symposium, Vol. 1, No. 1, pp. 18-21, 1996.

24. S. Targonski, R. Waterhouse, D. Pozar, Design of wide-band aperture-stacked patch microstrip antennas, in IEEE Transactions on Antennas and Propagation, Vol. 46, No. 9, pp. 1245-1251, 1998.

25. F. Croq, A. Papiernik, Large bandwidth aperture coupled microstrip antenna, in Electronics Letters, Vol. 26, No. 16, pp. 1293-1294, 1990.

26. N. Gupta, R. Tomar, P. Bhartia, A low-loss voltage-controlled analog phase-shifter using branchline coupler and varactor diodes, in ICMMT '07. International Conference on Microwave and Millimeter Wave Technology, pp. 1-2, 2007.

27. Impinj. Leading uhf gen 2 rfid technology innovator, 2012. URL http://www.impinj.com/.

28. J. Griffin, G. Durgin, Complete link budgets for backscatter-radio and RFID systems, in IEEE Antennas and Propagation Magazine, Vol. 51, No. 2, pp. 11-25, 2009.

\section{Author Biographies}

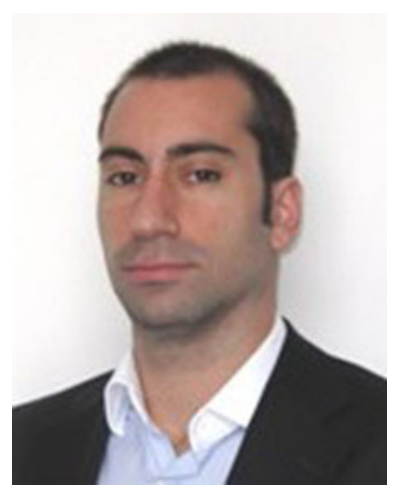

Antonios Bouzakis graduated from the Aristotle University of Thessaloniki in 2007 with a master's degree in mechanical engineering. Since 2008 he is with the Institute of Transport and Automation Technology as a research associate. His main scientific interests are in the field of indoor communication, location awareness, remote sensing and warehouse automation.

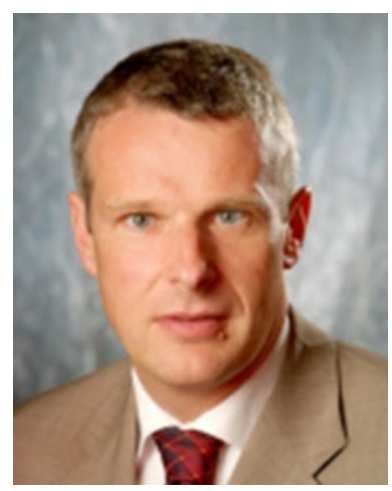

Professor Ludger Overmeyer studied electrical engineering at the Leibniz Universität Hannover and, subsequently, worked as research associate at the Laser Zentrum Hannover e.V. (LZH). After receiving his doctorate in mechanical engineering he was executive manager of research and development in the field of machine and plant manufacture for the semiconductor industry. Since 2002 he heads the Institute of Transport and Automation Technology (ITA) at the Leibniz Universität Hannover. In 2007 he became member of the management board of the IPH - Institute of Integrated Production Hannover (non-profit limited company). 